\title{
ATLAS Forward Proton Time-of-Flight Detector: LHC Run2 performance and experiences
}

\author{
T. Sykora* on behalf of ATLAS Collaboration \\ *Institute of Particle and Nuclear Physics \\ Faculty of Mathematics and Physics, Charles University \\ V Holešovičkách 2, 18000 Prague 8, Czech Republic \\ E-mail: tomas.sykora@mff.cuni.cz
}

\begin{abstract}
The performance of the ATLAS Forward Proton Time-of-Flight (ToF) Cherenkov detector is studied using the ATLAS data collected in 2017 during the LHC Run2. The detailed analysis of the results, including detector efficiency and time resolution of the ToF detector, is discussed. The detector construction and its expected performance based on beam test results are briefly summarized first. Operational experiences are presented. They include problems due to attempts to operate Micro-Channel Plate PhotoMulTipliers (MCP-PMTs) in the vacuum, leading to changes in the detector construction. In addition, a new MCP-PMT design was needed, as a result of the observed gain drop for high rates and its non-recoverability. Also, other hardware changes are presented - the effect of the replacement of glued crystal bars by monolithic ones, as well as the change of the MCP-PMT back-end electronics, based on simulation to reduce signal cross-talk resulting in further detector time resolution improvement. The second part of the document is devoted to the achieved time resolutions, $20 \pm 4$ ps and $26 \pm 5$ ps, of two installed ToF detectors. Despite their very low efficiencies (below 10\%) in major parts of the analyzed data, this represents a superb time resolution for detectors operating a few millimeters from the LHC beams. Based on the time resolution, this result characterizes the best ToF detector among those operated by the different LHC experiments in the forward regions during the LHC Run2. Finally, the possibility of reconstructing the z-coordinate of the ATLAS interaction region using the ToF detectors is shown.
\end{abstract}

Keywords: Timing detectors, Cherenkov and transition radiation, Performance of High Energy Physics Detectors 


\section{Contents}

1 AFP - physics and ToF subdetector 1

2 AFP - ToF detector design in 2017

3 AFP - ToF expected performance $\quad 4$

4 AFP - operational experience in $2017 \quad 6$

4.1 MCP-PMT operability in vacuum $\quad 7$

$\begin{array}{lll}4.2 & \text { MCP-PMT cross-talk } & 7\end{array}$

4.3 MCP-PMT \& high signal rates $\quad 8$

5 AFP ToF new design $\quad 11$

$\begin{array}{lll}5.1 & \text { New mechanical "Out-of-Vacuum" design } & 11\end{array}$

$\begin{array}{lll}5.2 & \text { LQBars } & 11\end{array}$

$6 \quad$ AFP ToF results in $2017 \quad 13$

$\begin{array}{lll}6.1 \text { Timing } & 13\end{array}$

$\begin{array}{lll}6.2 & \text { z-vertex reconstruction } & 14\end{array}$

$\begin{array}{lll}7 & \text { Conclusion } & 14\end{array}$

\section{AFP - physics and ToF subdetector}

The ATLAS [1] Forward Proton (AFP), one of the ATLAS Phase I upgrade projects [2], consists of four detector stations, two on each side of the ATLAS Interaction Point (IP), at $205 \mathrm{~m}$ and $217 \mathrm{~m}$. Each station is made of a single horizontal Roman pot housing the AFP detectors. The pots in near stations contain a four-plane Silicon 3D pixel detector (SiT). Both far pots contain the Time-ofFlight detector (ToF) behind another SiT tracker to measure the vertex position of double-proton final states. All detectors are operated at a few millimeters from the LHC beams. The schematic picture of the detector system is shown in Figure 1.

The use of ToF detectors is motivated by the suppression of the pile-up in central diffractive processes $\mathrm{p}+\mathrm{p} \rightarrow \mathrm{p}+\mathrm{X}+\mathrm{p}$, where $\mathrm{p}$ stands for a proton and $\mathrm{X}$ stands for any system produced during a collision, i.e. $\mathrm{X} \in\left\{\mathrm{H}, \mu^{+} \mu^{-}, \ldots\right\}$, via the reduction of the number of vertices, see Figure 2 . Naturally, the AFP ToF detector timing information can be combined with any other ATLAS ToF detector surrounding the ATLAS interaction region. 


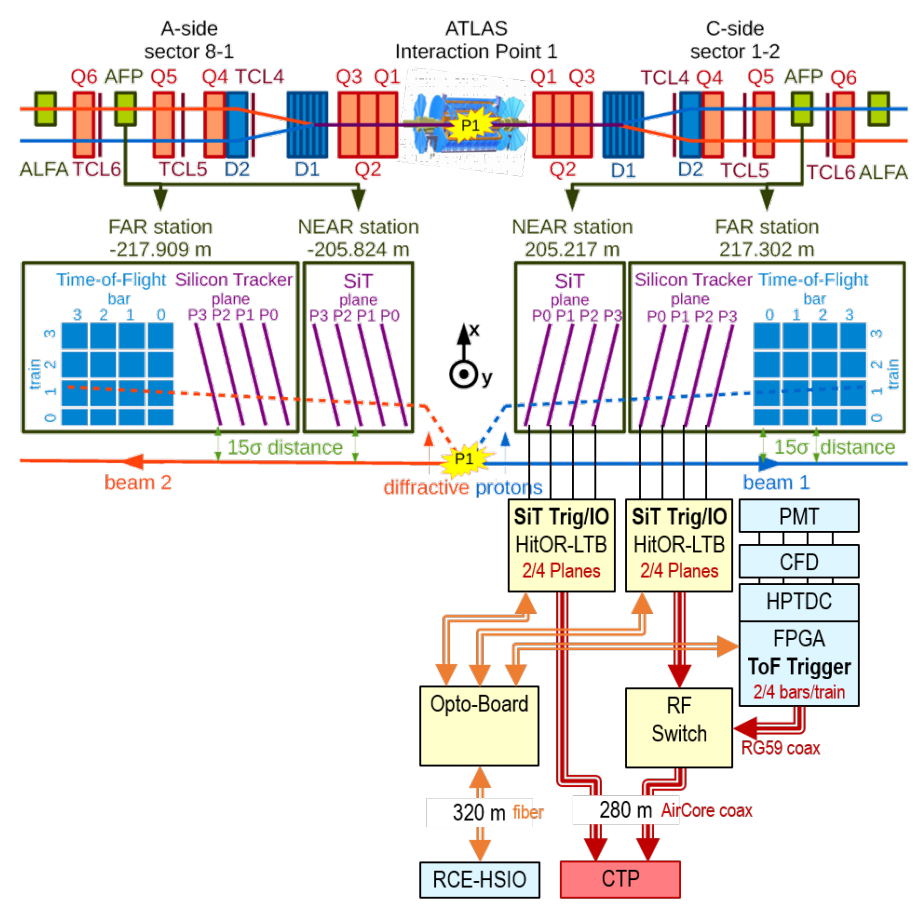

Figure 1. Schematic of the full AFP detector. A proton originating from a diffractive process passes through one of the AFP inner stations with the Silicon Tracking (SiT) detector and then through the corresponding outer AFP station with the SiT and ToF detectors. The ToF analog signal created by the (MCP-)PMT is then digitized by the Constant Fraction Discriminator (CFD) and time information is then built by the High Performance Time to Digital Converter (HPTDC). The schematic of the AFP trigger signal formation and readout (RCE) is shown too. The trigger signal is formed by both far SiT and ToF detectors, the decision about which one to use is made by the Radio Frequency (RF) switch and the signal is sent to the Central Trigger Processor (CTP). Copyright 2020 CERN for the benefit of the ATLAS Collaboration. CC-BY-4.0 license.

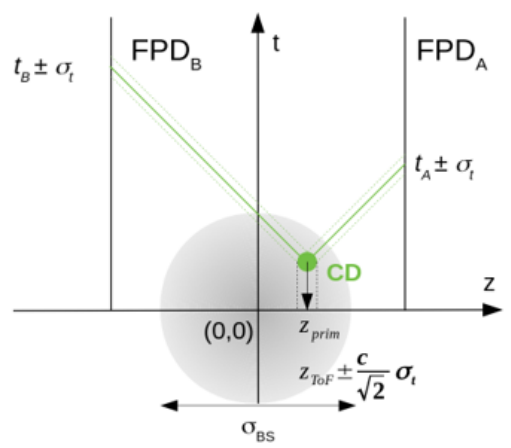

Figure 2. Graphic representation of the ToF detector principle: protons originating from a central diffractive event arrive into synchronized timing detectors $\mathrm{FPD}_{\mathrm{A}}$ and $\mathrm{FPD}_{\mathrm{B}}$. Based on the measured times and detector time resolutions the area $\mathrm{z}_{\mathrm{ToF}}$ around the position $\mathrm{z}_{\mathrm{prim}}$ of possible vertices is determined [3]. Copyright 2020 CERN for the benefit of the ATLAS Collaboration. CC-BY-4.0 license. 


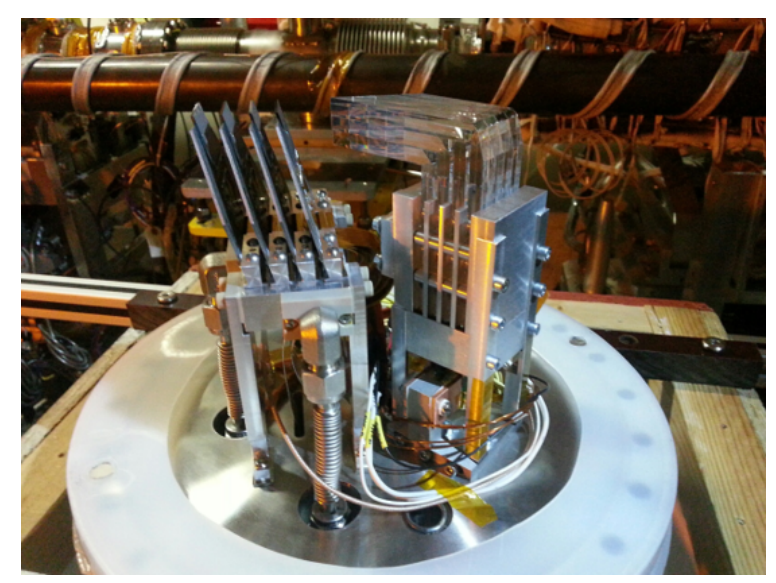

Figure 3. The AFP detector in the station at $217 \mathrm{~m}$ of the IP: a ToF quartz Cherenkov detector can be seen in the right part of the photo. L-shaped quartz bars (LQBars) serve as radiators and wave guides for Cherenkov photons. They are made of fused silica SK-1300, glued in the required L-shape using optical epoxy EPO-TEK 305 (glue) and are held in the aluminum holder. Cherenkov photons are received by the MCP-PMT located in the bottom part of the holder. On the left, the 4-plane SiT is located, mounted on its heat exchanger. Copyright 2020 CERN for the benefit of the ATLAS Collaboration. CC-BY-4.0 license.

\section{AFP - ToF detector design in 2017}

The design of the AFP ToF detector, shown in Figures 3 and 4, was originally supposed to address the following requirements:

1. resolution of $10 \mathrm{ps}$ ( $30 \mathrm{ps}$ in the first phase),

2. acceptance over the full range of the tracker detector,

3. near $100 \%$ efficiency,

4. segmentation for multi-proton timing,

5. high rate capability, $\sim 5 \mathrm{MHz} /$ segment,

6. Level 1 trigger capability,

7. radiation hardness.

The time resolution, using the 2017 detector design, could not reach 10 ps: the main limitations came from the geometry of LQBars ( $17 \mathrm{ps} / \mathrm{channel})[4,5]$, causing the spread in time of arrival of Cherenkov photons to the MCP-PMT due to their different wave lengths, and from the HPTDC ( 17-22 ps/channel) [6]. The latter limitation will be minimized by replacing the HPTDC by the picoTDC ( $<6$ ps/channel), planned in 2020.

Another potential limitation (the MCP-PMT behaviour at high signal rates) will be discussed in Section 4.3. The hardware limit of the HPTDC, $8 \mathrm{MHz} / \mathrm{segment}$, imposed by its construction, was not reached in the LHC Run2. 


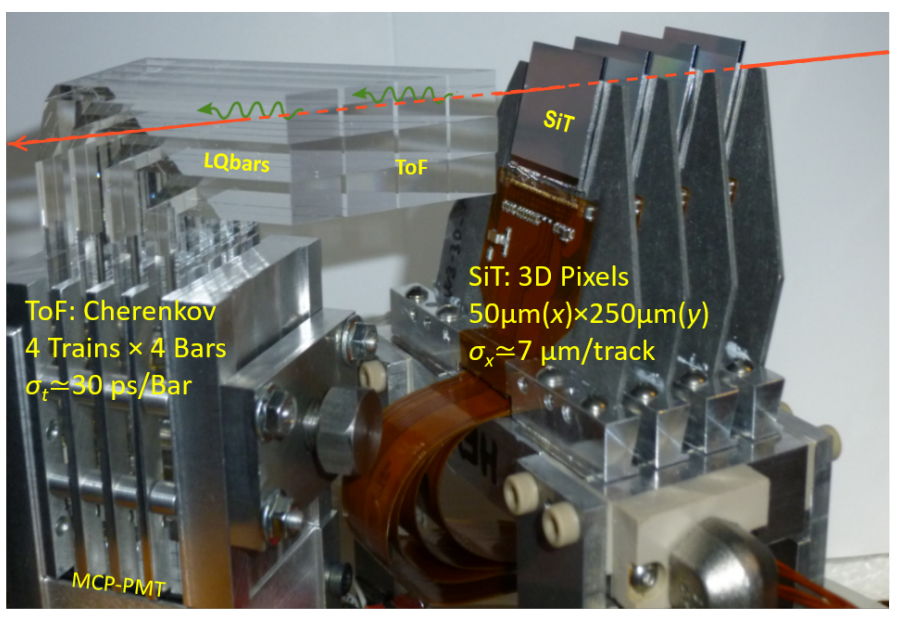

Figure 4. Naming conventions (LQBars, trains) used in the description of the ToF detector. The passage of a proton through the AFP silicon tracker then through the ToF detector is depicted. Trains are enumerated from 0 to 3, LQBars in each train are labeled from A to D; in case two MCP-PMTs are used in a measurement, the numbering range of trains is enlarged to 0-7. Copyright 2020 CERN for the benefit of the ATLAS Collaboration. CC-BY-4.0 license.

\section{AFP - ToF expected performance}

Based on the series of beam tests performed by the AFP ToF group at the CERN SPS in the years 2014-2018 using the setup configuration shown in Figure 5, the expected time resolution of the ToF detector without the HPTDC and synchronization clock contributions (measured by the oscilloscope) is in the case of one LQBar (i.e. one channel) $\sim 20 \mathrm{ps}$ and in the case of the full train $\sim 14$ ps, see Figure 6. In both cases the contribution of the trigger is subtracted.

The contributions of each ToF component to the time smearing are summarized in Table 1.

Table 1. ToF components and their contributions to the detector time resolution per channel.

\begin{tabular}{ll}
\hline part & contribution [ps] \\
\hline LQBar & 17 \\
MCP-PMT & $<10^{*}$ \\
PAa/b/c (preamplifiers, 3 layers) & $3-4$ (each) \\
CFD & 5 \\
HPTDC & $15-17$ (up to 25 before a calibration) \\
picoTDC & $<6$ \\
reference clock & 6 \\
\hline
\end{tabular}

*In case of signal rate $<1 \mathrm{MHz}$.

The time measurements obtained from different channels in a train are not independent from each other, see Figure 7. Indeed, the final time resolution does not scale as the square-root of the number $N$ of channels used in the time measurement (in our case each train has 4 LQBars and $N=4$ ). This is due to the cross-talk caused by the MCP-PMT back-end electronics. This point is addressed later in Section 4.2. 


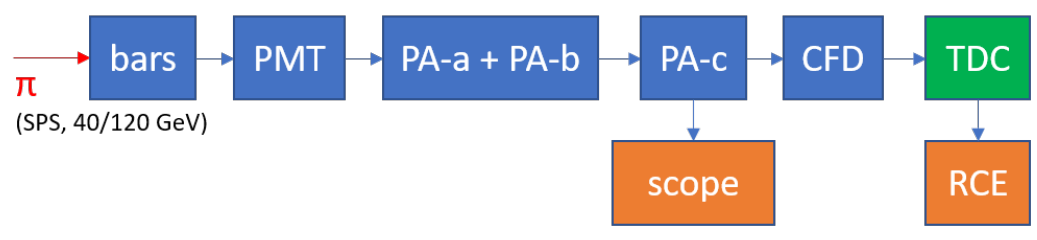

Figure 5. Schematic of the AFP ToF beam test setup at the CERN SPS. A MIP particle, e.g. a pion, is passing through the bars, generating Cherenkov light, which is transformed into a pulse by the PMT. The amplitude of the pulse is multiplied by the chain of preamplifiers PAa, PAb and PAc and then either recorded by an (oscillo)scope or digitized by the CFD. The time information is built by the (HP)TDC and is recorded by the AFP readout system RCE. Copyright 2020 CERN for the benefit of the ATLAS Collaboration. CC-BY-4.0 license.
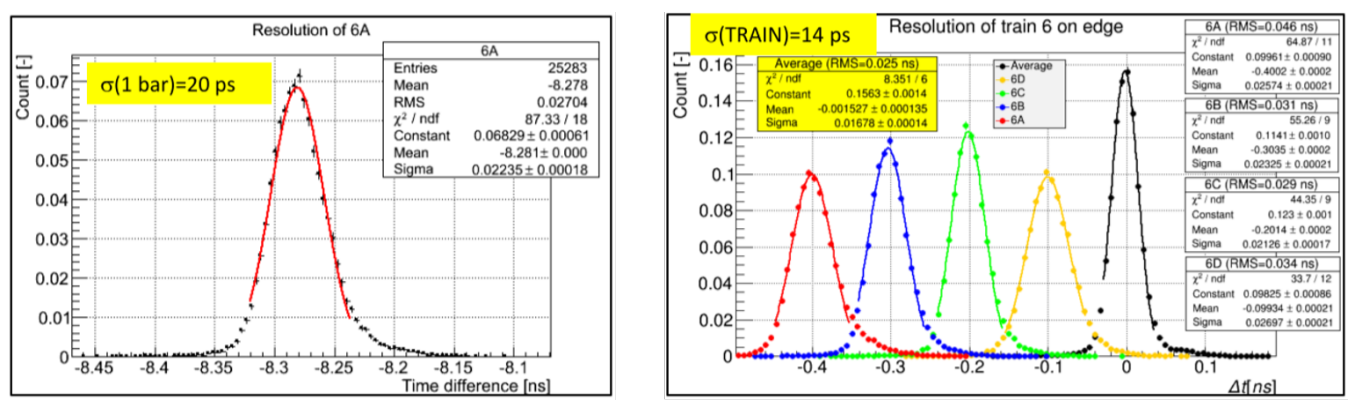

Figure 6. Distributions of the measured time w.r.t. the used trigger (SiPM) in case of (left) a single LQBar, (right) a full train (= 4 LQBars, see Figure 4). In both cases the contribution of the trigger to the time resolutions, $\sigma(\mathrm{X})$, is subtracted. This measurement was done by using an oscilloscope, see Figure 5, i.e. there are no contributions from the CFD and the HPTDC components [7].

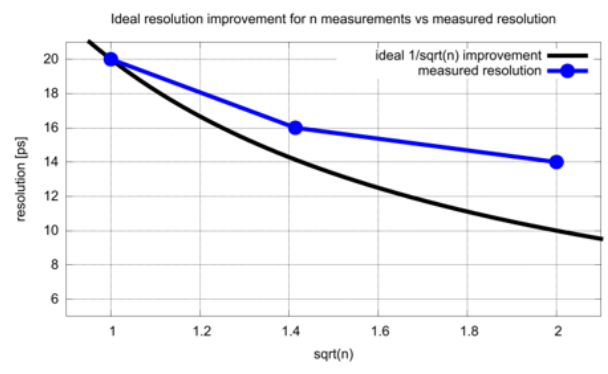

Figure 7. Ideal (black) and measured (blue) curves showing the time resolution of a detector as a function of the number of used channels. The time resolution is expected to vary as $\sim \sqrt{N}$. The data from [7] are plotted. Copyright 2020 CERN for the benefit of the ATLAS Collaboration. CC-BY-4.0 license.

The time distribution of the full system, including contributions of the CFD and HPTDC but without the sychronization clock, is shown in Figure 8. The time resolution, after subtraction of the trigger contribution, is $\sim 14$ ps. 


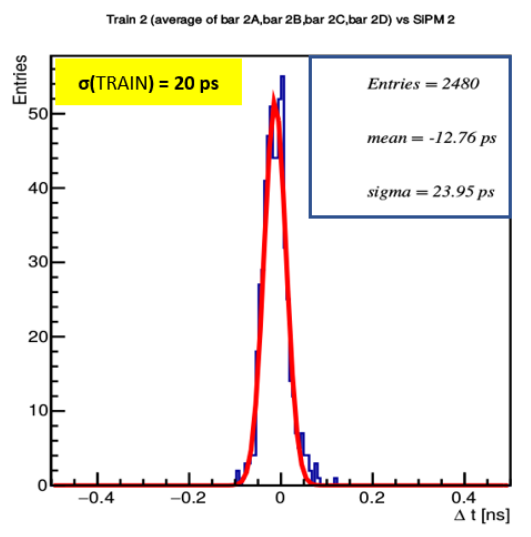

Figure 8. Time distribution of the full system, including contributions of the CFD and HPTDC but without the sychronization clock [8].

\section{AFP - operational experience in 2017}

The AFP ToF detector was installed during the 2016-2017 LHC winter shutdown and the first timing data were taken in May 2017. The ToF detector was operated until the beginning of the heavy ions data taking period at the end of same year.

After the proper timing-in procedure (trigger and data synchronization) the expected patterns, known from the numerous beam tests, were not observed. When trying to find the reason, e.g. by comparing the number of tracks recorded by the SiT detector with the number of events recorded by the ToF detector, it was found that the efficiency of the ToF detector was very low [3].

The reasons for missing patterns were understood only later (at the end of the data taking period): both MCP-PMTs were operated with too low High Voltage (HV) leading to low gain. While the target gain was $5 \times 10^{4}$, the actual gain at $2.0 \mathrm{kV}$ was $\sim 1-2 \times 10^{4}$ leading to a CFD threshold inefficiency. Simultaneously, despite operating the MCP-PMTs at very low gain, the life times of both MCP-PMTs $\left(\leq 1 \mathrm{C} / \mathrm{cm}^{2}\right)$ were exceeded by an estimated factor 3-6, which also contributed to the gain deterioration of both MCP-PMTs.

Problems observed with the ToF efficiency in 2017 were understood but subsequent issues with the operation of MCP-PMTs in the secondary vacuum of the pots prohibited the re-installation of the ToF detector in 2018. The problems with MCP-PMTs started one week before the installation (spring 2017): one of the MCP-PMTs started to trip repeatedly when operated in the secondary vacuum ( 30 mbar). It was assumed that the trips were caused by a failing MCP-PMT insulation at the back-end side with powering HV cables and electronics. The attempt to use an additional urethane lacquer as an insulating layer was not successful. The situation was solved by the delivery of another MCP-PMT (of the different type) by the original provider, Photonis Defence Inc. Both MCP-PMTs were then operated without any observed trip before their installation and during their operation in the LHC tunnel. Both MCP-PMTs were non-ALD (Atomic Layer Deposition) coated and their lifetimes were expected to be of the order of $0.6-1 \mathrm{C} / \mathrm{cm}^{2}$, which corresponded, depending on the number of bunches and pile-up, to 2-4 months of operation. 

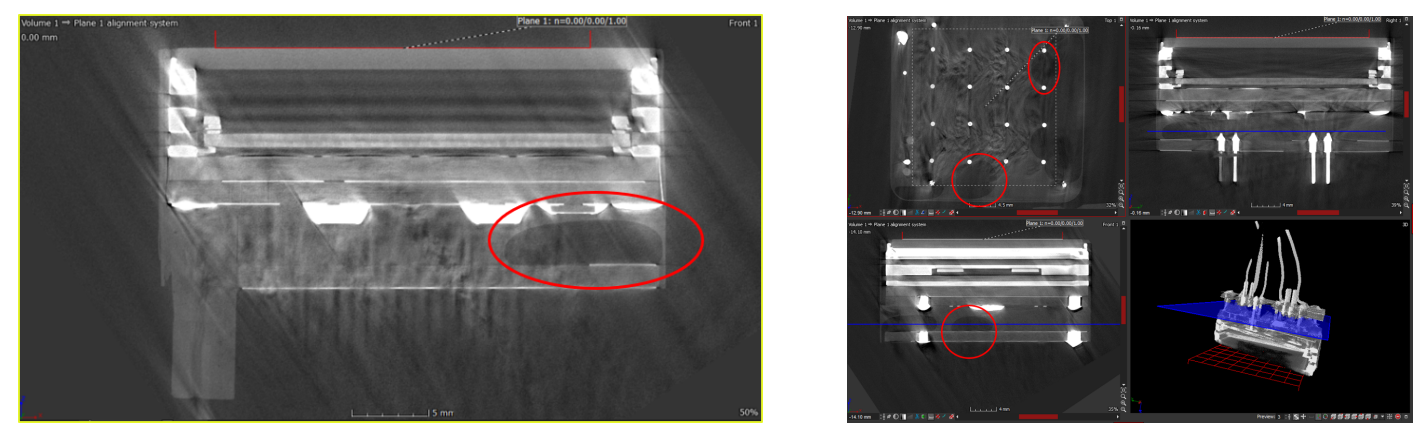

Figure 9. 3D X-ray scan of one of the Photonis XPM85212 MCP-PMTs in order to identify eventual weak points leading to repeated failures of MCP-PMTs when operated in vacuum. Some of the air bubbles are depicted by red ovals. Copyright 2020 CERN for the benefit of the ATLAS Collaboration. CC-BY-4.0 license.

\subsection{MCP-PMT operability in vacuum}

The plan to re-install the ToF detector after the winter break in 2018 was unsuccessful due to repeated failures of the MCP-PMTs in the vacuum during their tests. There were in total 3 attempts (after the LHC winter break, technical stops 1 and 2) to install the AFP ToF detectors, each time unsuccessful for the same reason - trips of the MCP-PMTs in vacuum. The MCP-PMTs were scanned using a 3D X-ray facility at CERN, which led to several observations: air bubble gaps in the MCP-PMT potting, different design of electronics with respect to the one agreed with the provider, and potentially weak points in the HV powering scheme which can lead to discharges when the MCP-PMT was operated in vacuum.

The plan was to refurbish 3 existing MCP-PMTs by potting them at CERN. However, despite the original success (the first of the refurbished MCP-PMT was tested without problems for $\sim 2$ months), a proper way for a safe potting of MCP-PMTs was not found at the end (another MCP-PMT was re-potted twice but kept tripping). In particular, the area close to the MCP-PMT cathode (under the MCP-PMT window) remained critical.

\subsection{MCP-PMT cross-talk}

As mentioned earlier, timing information from different MCP-PMT channels was found not to be independent due to the cross-talk between MCP-PMT channels. There are different types of crosstalk: an optical one, charge sharing and one due to the construction of the MCP-PMT back-end circuitry. Actually, only the last one leads to the correlation between the channels, violates the $\sqrt{N}$ scaling and worsens the time resolution of the detector.

Knowing the MCP-PMT construction, see Figure 10, its dimensions and the estimated properties of the used material, a corresponding SPICE model was made for studies aiming to understand different behaviours of tubes measured in last years. For instance, some of them had modified backend electronics (removed resistance elements) which lead to much higher cross-talk and "ringing" of such photomultipliers.

A comparison of tube simulations, with an input signal in one of the MCP-PMT channels, was made with data, see the left part of Figure 11. The ability to simulate the response of a 

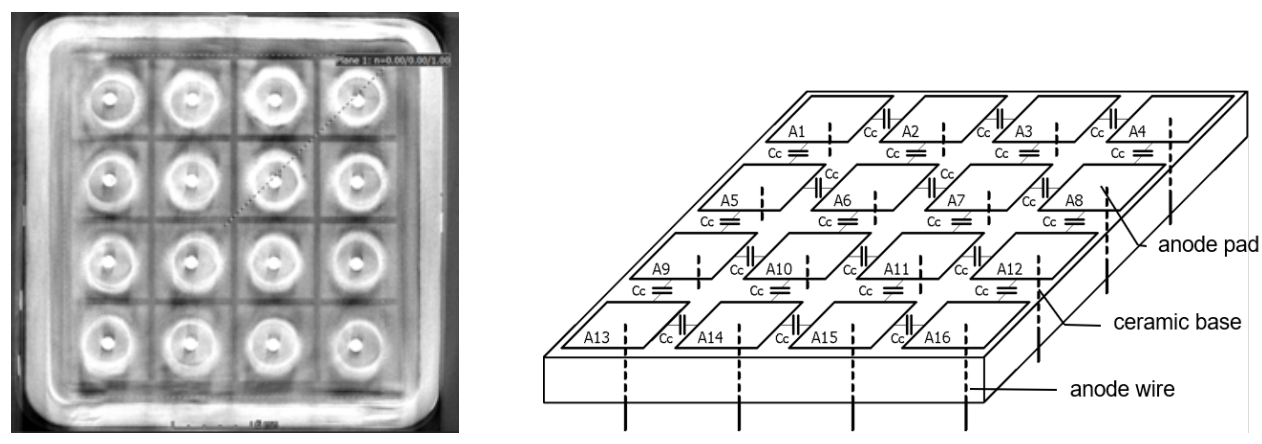

Figure 10. (left) Anodes (projection) of a MCP-PMT as seen at the CERN 3D X-ray facility and (right) a schematic of the model implemented in SPICE for MCP-PMT simulations. Copyright 2020 CERN for the benefit of the ATLAS Collaboration. CC-BY-4.0 license.

photomultiplier to the signal in a full train is shown in the right plot in Figure 11, where cross-talk waveforms for four neighboring channels are shown.
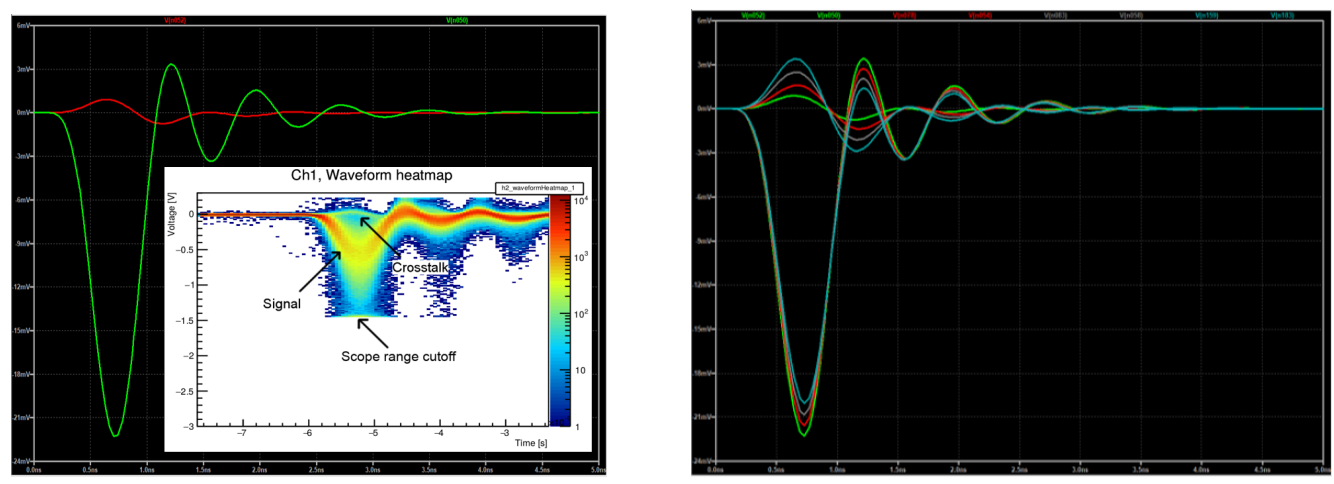

Figure 11. (left) Simulated output signal and cross-talk waveforms in the case of incoming signal in one of the MCP-PMT channels and corresponding measured signal/cross-talk waveforms in an oscilloscope (embedded window); (right) simulated output signal and cross-talk waveforms in case of an input signal in all MCP-PMT train channels. Copyright 2020 CERN for the benefit of the ATLAS Collaboration. CC-BY-4.0 license.

\subsection{MCP-PMT \& high signal rates}

The fact that the MCP-PMT loses its gain when the signal rate increases is well known (MCP-PMT saturation). It was not expected that this effect would cause the observed low efficiency of the AFP ToF detector during the first, low pile-up, runs in 2017. Nevertheless the testing of MCPPMT using high signal rates were done at the University of Texas, Arlington [9]. Two units of two manufacturers, Photonis and Hamamatsu, were tested, see Figures 12 and 13. The timing properties of Photonis MCP-PMTs start to deteriorate at a signal rate of $4 \mathrm{MHz}$. In the case of Hamamatsu MCP-PMTs, it starts already at the level of $1 \mathrm{MHz}$.

A new, surprising, effect was observed: all tested tubes lost their gain for quite a long time after being exposed to the high signal rates. This observation was made also by ALICE group [10] and the effect was confirmed also for an ALD coated tube produced by Photek Ltd. A small study 

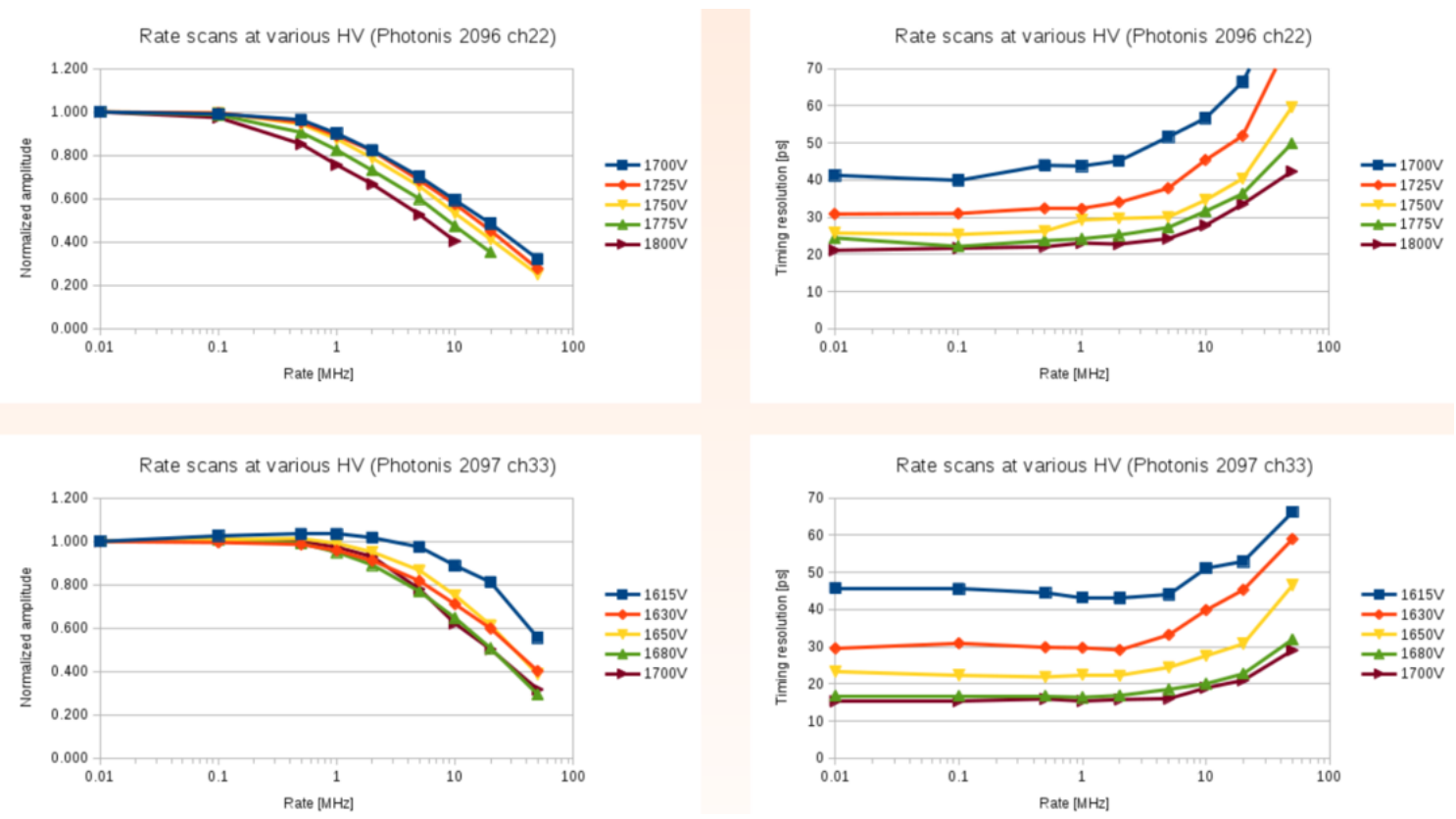

Figure 12. (left column) Dependency of the normalized amplitude on the signal rate and different values of applied HV for two Photonis XPM85212 MCP-PMTs. Information on the tube serial numbers appears in the legend; (right column) dependency of the time resolution in ps on the signal rate and different values of applied HV for the same two photomultipliers [9].
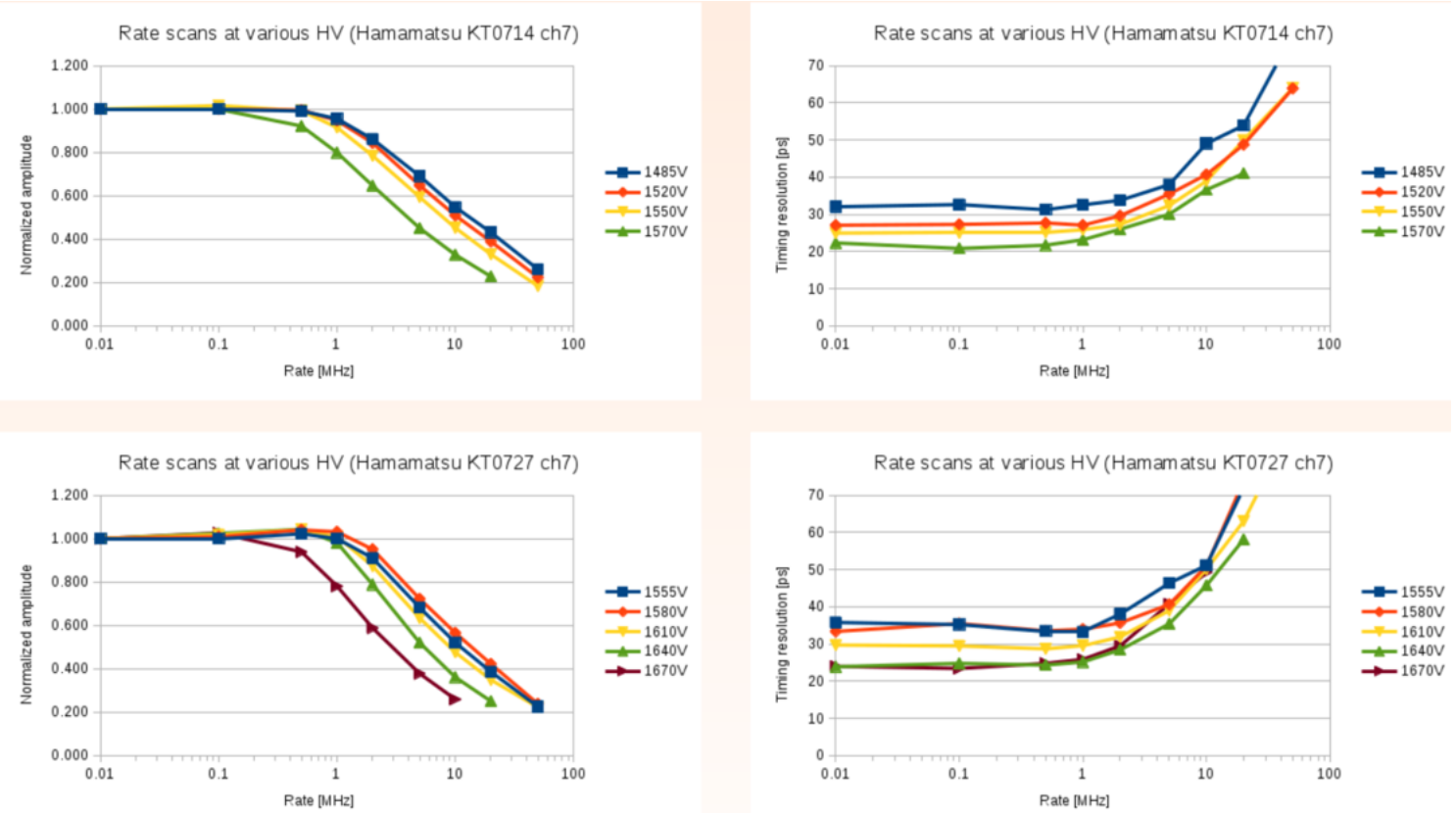

Figure 13. (left column) Dependency of the normalized amplitude on the signal rate and different values of applied HV for two Hamamatsu R10754-07-M16 MCP-PMTs. Information on the tube serial numbers appears in the legend; (right column) dependency of the time resolution in ps on the signal rate and different values of applied HV for the same two photomultipliers [9]. 


\begin{tabular}{|c|c|c|c|}
\hline Device \# & 1 & 2 & 3 \\
\hline MCP-PMT type & R10754-07-M16 & PMT253 & XP85002/FIT-O \\
\hline Manufacturer & Hamamatsu & Photek & Photonis \\
\hline $\begin{array}{c}\text { ALD-coating of } \\
\text { the MCPs }\end{array}$ & yes & yes & no \\
\hline $\begin{array}{l}\text { Resistance of the } \\
\text { MCP stack }\end{array}$ & $40 \mathrm{M} \Omega$ ? & $15.5 \mathrm{M} \Omega$ & $14 \mathrm{M} \Omega$ \\
\hline Total bias voltage & $1600 \mathrm{~V}$ & $3000 \mathrm{~V}$ & $1285 \mathrm{~V}$ \\
\hline $\begin{array}{l}\text { Voltage across the } \\
\text { MCP stack }\end{array}$ & & $1704 \mathrm{~V}$ & $1018 \mathrm{~V}$ \\
\hline $\begin{array}{l}\text { Sensitive area of } \\
\text { an individual } \\
\text { readout channel }\end{array}$ & $5.3 \times 5.3 \mathrm{~mm}^{2}$ & $6.6 \times 6.6 \mathrm{~mm}^{2}$ & $26.5 \times 26.5 \mathrm{~mm}^{2}$ \\
\hline Serial number & КT0714 & A1171005 & 9002140 \\
\hline
\end{tabular}

Figure 14. Summary table of MCP-PMTs (and their properties) used in the study of their responses to high signal rates [11].
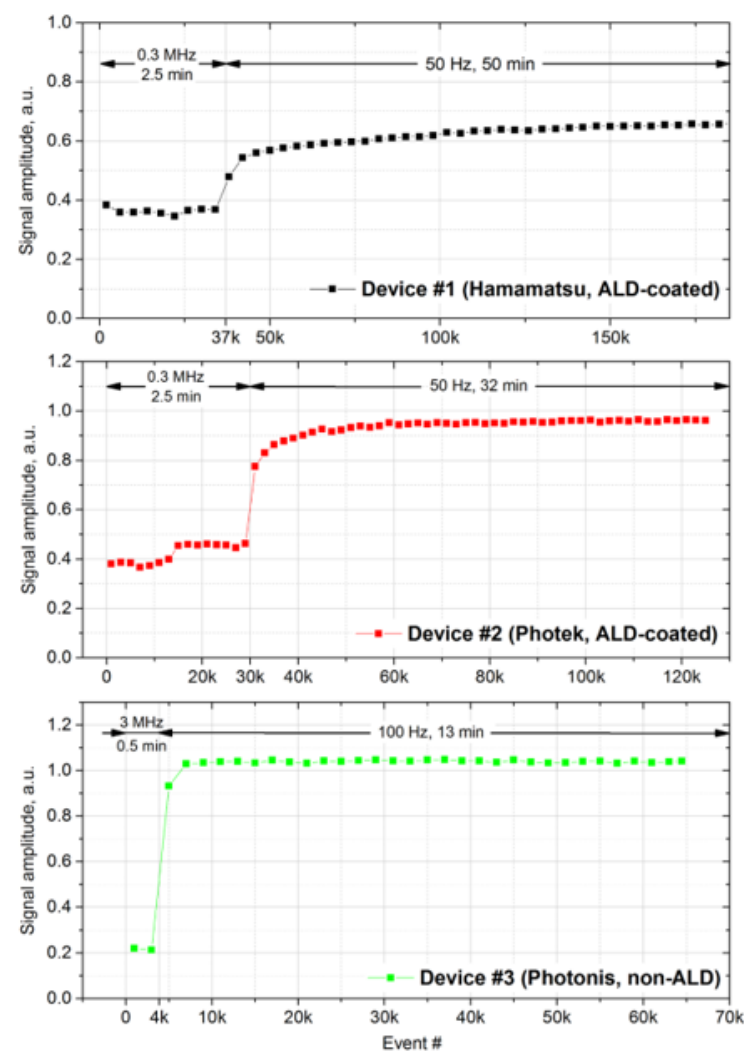

Figure 15. Relative signal value of the tested MCP-PMTs normalized to that of the reference PMT versus integrated number of counts (digitized pulse waveforms) [11].

[11] comparing tubes, with properties summarized in Figure 14, was made. The measurements of the recoverability time for each MCP-PMT is shown in Figure 15. The effect of long-time gain loss due to their exposure to the high signal rate was not observed for non-ALD MCP-PMTs.

Although experts believe that the issue comes from charging of the MCP-PMT ALD layer, see e.g. [9], a simulation model describing this effect has not been developed yet. 


\section{AFP ToF new design}

The fact that a few millimeter gap between quartz bars and an MCP-PMT cathode window did not deteriorate timing has led to a possibility to introduce a secondary vacuum separator in the form of a window made of the same material as LQBars (SK-1300). The beam test measurements were in very good agreement with simulations and confirmed that the separation window does not significantly deteriorate timing. The simulations were done for different window thicknesses (1-3 $\mathrm{mm})$. For the $3 \mathrm{~mm}$ thick window, which was selected to make the design robust enough, the timing was worsened by less than 1 ps (for the full train) and the cross-talk amplitude increased by maximally $25 \%$ (per channel), which is safely coverable/removable by the corresponding increase of channel CFD thresholds. The new design has the following advantages:

1. no trip of an MCP-PMT was observed when it was operated under air pressure,

2. full warranty for the MCP-PMT (currently there is no producer of MCP-PMTs providing warranty for operation in vacuum),

3. possibility to lower the MCP-PMT resistance, which should help with expected high rates (saturation),

4. cooling in air is better than in vacuum,

5. no need for special feedthroughs - both for HV and signal lines,

6. possibility to access and exchange, if needed, MCP-PMTs or preamplifiers very quickly, without breaking the secondary vacuum.

In relation with the new design the construction of the first stage of preamplifiers had to be changed. Two different constructions were prepared and will be tested in the laboratories and beam tests before a decision about which one to use is made.

\subsection{New mechanical "Out-of-Vacuum" design}

As stated in the introduction above, after preliminary studies that proved the feasibility of the new concept, the detailed design and construction of the mechanical part was made. It happened in several iterations, included discussions with the LHC experts, and was accompanied by tests of the vacuum tightness of the prototype and, also, its reliability in case of sudden over-pressure in the LHC tunnel. A comparison of the old and new mechanical design is shown in the upper part of Figure 16, the final solution is illustrated in the lower part.

\subsection{LQBars}

The construction of the LQBars was also changed. New bars will be made of one piece of material (monolithic). The change removes the usage of glue which represents a weak point of the LQBars construction due to its limited radiation hardness (eventually affecting also the bond strength) and restricted transmissivity, see the left part of Figure 17. On the other hand these points were not critical and on the contrary, due to the technological limitations in the production of monolithic bars, the minimal train thickness increased. 


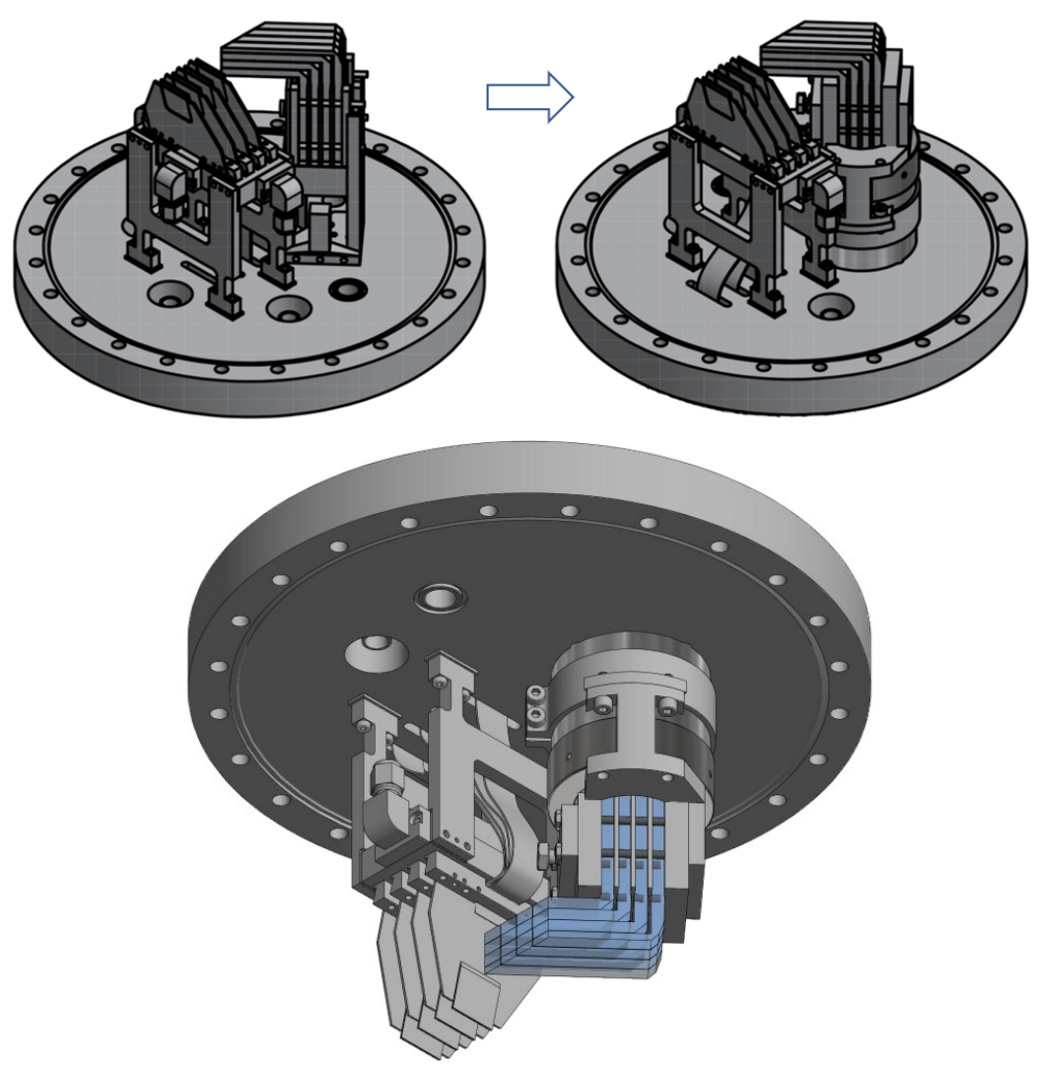

Figure 16. (upper) Comparison of old and new designs of the ToF detector; (lower) view of the (new) flange side with exposed Si tracker modules and ToF LQBars (in transparent blue color). Copyright 2020 CERN for the benefit of the ATLAS Collaboration. CC-BY-4.0 license.

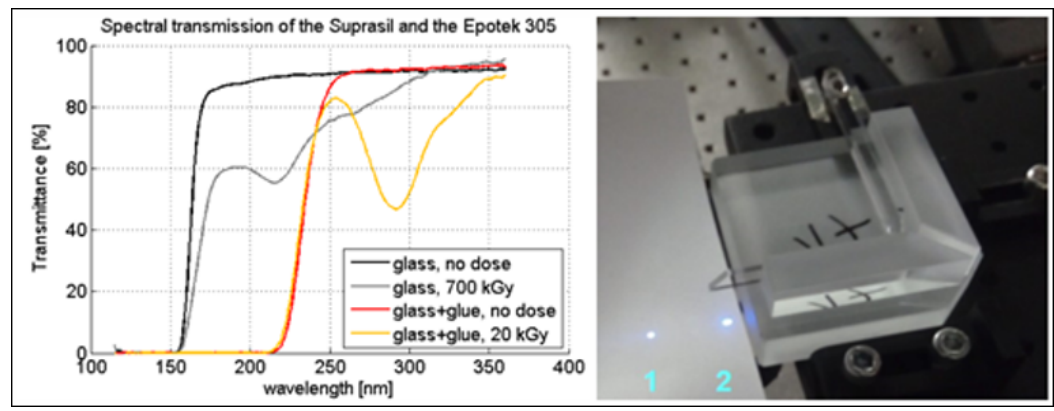

Figure 17. (left) Transmission spectra of the SK-1300 material used for the production of LQBars and of EPO-TEK 305 (glue) before and after irradiation (including Fresnell losses), (right) splitting of a laser beam observed during transmittance measurements of irradiated bars [12]. 


\begin{tabular}{|c|c|c|c|c|c|c|}
\hline & \multicolumn{3}{|c|}{ Glued bars } & \multicolumn{3}{|c|}{ Solid bars } \\
\hline Bar & $\begin{array}{l}\text { gain } 5.4 \cdot 10^{4} \\
\text { (HV } 2100 \mathrm{~V})\end{array}$ & $\begin{array}{l}\text { gain } 7.6 \cdot 10^{4} \\
\text { (HV } 2150 \mathrm{~V})\end{array}$ & $\begin{array}{l}\text { gain } 1.1 \cdot 10^{5} \\
\text { (HV } 2200 \mathrm{~V})\end{array}$ & $\begin{array}{l}\text { gain } 3.8 \cdot 10^{4} \\
(\mathrm{HV} 2050 \mathrm{~V})\end{array}$ & $\begin{array}{l}\text { gain } 5.4 \cdot 10^{4} \\
\text { (HV } 2100 \mathrm{~V})\end{array}$ & $\begin{array}{l}\text { gain } 7.6 \cdot 10^{4} \\
\text { (HV 2150 V) }\end{array}$ \\
\hline $2 \mathrm{~A}$ & 40 & 39 & 35 & 49 & 46 & 42 \\
\hline $2 B$ & 40 & 38 & 36 & 47 & 43 & 40 \\
\hline $2 \mathrm{C}$ & 39 & 36 & 32 & 45 & 43 & 40 \\
\hline $2 \mathrm{D}$ & 38 & 36 & 34 & 44 & 41 & 38 \\
\hline
\end{tabular}

\begin{tabular}{|c|c|c|c|c|c|c|c|}
\hline & & \multicolumn{3}{|c|}{ Glued bars } & \multicolumn{3}{c|}{ Solid bars } \\
\hline HV [V] & Gain [-] & edge & $5 \mathrm{~mm}$ & $9 \mathrm{~mm}$ & edge & $5 \mathrm{~mm}$ & $9 \mathrm{~mm}$ \\
\hline 2050 & $3.8 \mathrm{E}+4$ & - & - & - & $24 \pm 2$ & $26 \pm 2$ & $26 \pm 2$ \\
\hline 2100 & $5.4 \mathrm{E}+4$ & $21 \pm 2$ & $24 \pm 2$ & $25 \pm 2$ & $\mathbf{2 4 \pm 2}$ & $\mathbf{2 6 \pm 2}$ & $\mathbf{2 6} \pm 2$ \\
\hline 2150 & $7.6 \mathrm{E}+4$ & $\mathbf{2 4} \pm 2$ & $\mathbf{2 4} \pm 2$ & $\mathbf{2 6} \pm 2$ & $24 \pm 2$ & $26 \pm 2$ & $26 \pm 2$ \\
\hline 2200 & $1.1 \mathrm{E}+5$ & $20 \pm 2$ & $25 \pm 2$ & $27 \pm 2$ & - & - & - \\
\hline
\end{tabular}

Figure 18. (upper) Timing resolution of single bars in ps (uncertainty \pm 2 ps) for the amplitude threshold of $-150 \mathrm{mV}$ at the distance of $5 \mathrm{~mm}$ from the edge; (lower) Timing resolution of train $\mathbf{2}$ in ps composed of glued and monolitic bars for the amplitude threshold of $-150 \mathrm{mV}$ at the distance of $5 \mathrm{~mm}$ from the ToF radiator edge [12]. Copyright 2020 CERN for the benefit of the ATLAS Collaboration. CC-BY-4.0 license.

The selected results, obtained during the beam test at DESY in 2019, are summarized in Figure 18 [12]. It was surprising that the time resolution in case of monolithic bars was worse by $3 \pm 1 \mathrm{ps}$ in average, as the amount of light, due to the removal of the optical glue, increased by a factor 1.6 and a small improvement of the time resolution was thus expected.

A possible explanation is based on the effect of the Cherenkov light dispersion: the wavelength range of accepted photons spreads (when the glue is not present) down to $160 \mathrm{~nm}$ where the index of refraction of the fused silica quickly rises. This leads to a widening of the light pulse on the photocathode of the photomultiplier and distortion of the output signal. However, this effect was not observed in simulation.

\section{AFP ToF results in 2017}

Selected runs from 2017 were analyzed to check possible dependencies of the detector inefficiency on the number of bunches in the LHC beams, the size of pile-up and the ToF HV setting.

\subsection{Timing}

The time resolution of each ToF channel, extracted from two low pile-up runs $(\mu \sim 2)$ is shown in Figure 19 [3]. The results are in a good agreement with beam test results; note however that "diffractive" protons in the LHC tunnel are passing far from the edges of radiators, the amount of Cherenkov light in corresponding LQBars is smaller and the time resolution is worse than presented e.g. in Figure 6. The observed pattern showing improved resolution as a function of the channel (independently of a train/station) is explained by the increasing amount of light in corresponding LQBars (due to the detector geometry): the light from channel A leaks into the channels B and C, the light from channel $\mathrm{B}$ to $\mathrm{C}$ and $\mathrm{D}$, etc. The train $\mathrm{A}$ gets no additional light, the channel $\mathrm{B}$ gets light just from the channel A. However, there is no explanation yet why the resolution of channel D is worse than that of channel C. 


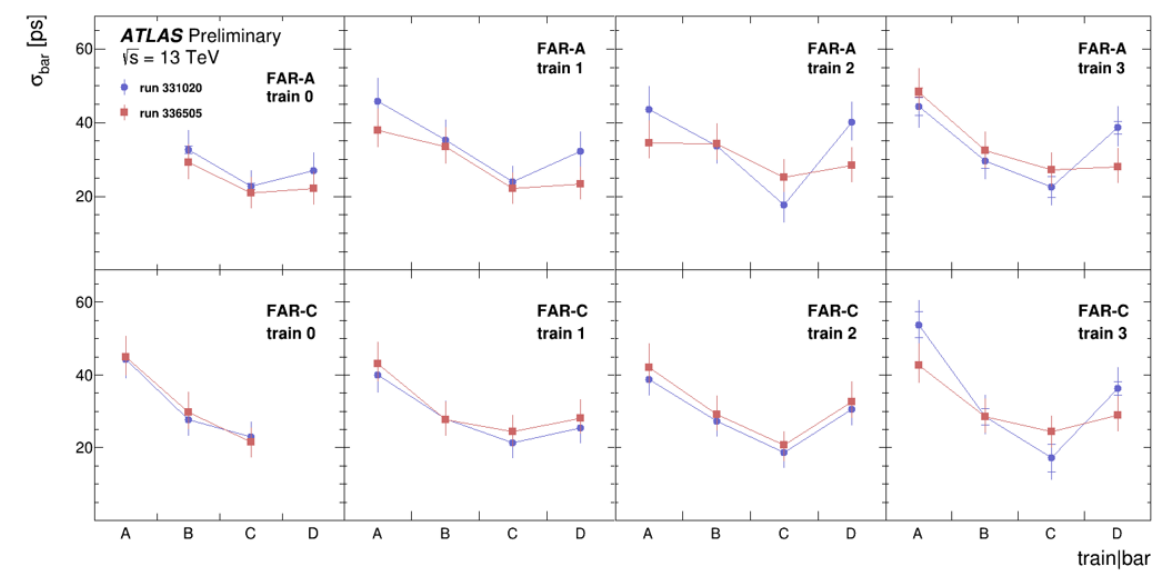

Figure 19. Time measurement resolutions of single ToF channels extracted from AFP calibration stream runs 331020 and 336505. The full error bars represent the statistical uncertainties of the resolution fits and the systematic uncertainties (time measurement correlations between channels and calibrations of the time measurement in the channels) added in quadrature. The time resolutions are extracted from the widths of the distributions of time differences $\Delta_{t_{\mathrm{ij}}}=t_{\mathrm{i}}-t_{\mathrm{j}}$ within a single train, where $\mathrm{i}$ and $\mathrm{j}$ denote channel numbers [3]. Copyright 2020 CERN for the benefit of the ATLAS Collaboration. CC-BY-4.0 license.

\section{2 z-vertex reconstruction}

A very interesting study of the $\mathrm{z}_{\mathrm{ATLAS}}-\mathrm{z}_{\mathrm{ToF}}$ distribution was done [3] and is illustrated in Figure 20 . The distribution was obtained by random mixing of times measured by the AFP ToF detector in either station and the $\mathrm{z}$ ATLAS values which do not originate in the same collision event, see Figure 2.

The expected resolution of the ToF detector, $\sigma_{\text {expected }}^{\text {ToF }}$, used in the study was obtained from the known single-channel resolutions, see Figure 19, convoluted with the actual channel-hit-patterns observed in the data in the 'no $\mathrm{N}_{\mathrm{vtx}}$ cut' scenario.

The shape of the distribution (narrow peak) provides a hint of the presence of the (central diffraction) signal $p+p \rightarrow p+X+p$ (see Section 1). Also, the width of the distribution is, within errors, in agreement with ATLAS measurements done using its Inner Detector.

\section{Conclusion}

The AFP ToF detector was installed and took data in 2017. The achieved timing performance was very good, despite very low efficiencies (which affected timing), and was in good agreement with beam test results. Due to the repeated failures of MCP-PMTs in vacuum the AFP ToF detector was not installed in 2018 and its new design, with MCP-PMTs placed out of the detector secondary vacuum, was prepared and approved. The newly designed construction of the MCP-PMT back-end electronics (to suppress cross-talk) was developed, successfully tested in 2020 and will be used in the construction of the new MCP-PMTs planned for the LHC Run3. The new sample of monolithic (glue-less) LQBars was tested, and a comparison with the original glued bars was done. Full sets for the LHC Run3 are in production now. As part of the new, out-of-vacuum, ToF design the simplified signal and powering paths will be tested with beam in 2020. The prototype of picoTDC was tested at the DESY beam test facility in 2019, further tests are planned in 2020. The new AFP ToF based 


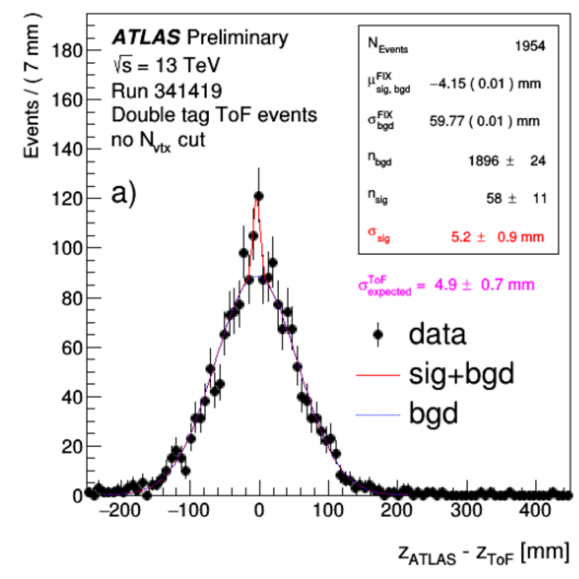

Figure 20. The distributions of $z_{A T L A S}-z_{T o F}$ measured in events with ToF signals on both sides of the interaction region in run 341419, where $\mathrm{z}_{\text {ATLAS }}$ is the primary vertex z-position reconstructed by ATLAS. The $\mathrm{z}_{\mathrm{ToF}}$ is obtained as $\mathrm{z}_{\mathrm{ToF}}=-\mathrm{c} / 2 \Delta(\mathrm{t})$, where $\Delta(\mathrm{t})$ is difference in proton arrival times in $\mathrm{A}$ and $\mathrm{C}$ far stations of the AFP measured by ToF. The distribution shown corresponds to ATLAS data containing a reconstructed primary vertices together with coincidence of signals in both ToF detectors in case of the scenario with no restriction on the number of the reconstructed verteces ('no $\mathrm{N}_{\mathrm{vtx} \text { cut }}$ ') [3]. Copyright $2020 \mathrm{CERN}$ for the benefit of the ATLAS Collaboration. CC-BY-4.0 license.

trigger module was partially tested in 2019; testing is ongoing and it will be finalized in a beam test in 2020. The installation of the modified and upgraded AFP ToF detector for the LHC Run3 is planned during the summer 2020. The most critical and still open question is whether the new type (R2D2) of MCP-PMTs, in preparation thanks to the collaboration of Arradiance with Photonis, will be possible to operate up to the signal rate of $20 \mathrm{MHz} / \mathrm{cm}^{2}$. The answer should be known by the end of 2020 .

The analysis of the 2017 LHC Run2 data have shown the good agreement with beam test results and confirmed the potential of the AFP ToF detector to achieve a time resolution of $15 \mathrm{ps}$ in the LHC Run3, assuming a good behaviour at high signal rate for the new MCP-PMTs.

\section{Acknowledgments}

The measurements leading to the presented results have been performed at the LHC at CERN, at the SPS Test Beam Facility at CERN, at the Test Beam Facility at DESY Hamburg (Germany), the member of the Helmholtz Association (HGF) and at the University of Texas at Arlington. The author gratefully acknowledges the support from the projects LM2015056 (CANAM) and LTT17018 (Inter-Excellence) of the Ministry of Education, Youth and Sports of the Czech Republic.

\section{References}

[1] ATLAS Collaboration, The ATLAS Experiment at the CERN Large Hadron Collider, JINST 3 (2008) S08003.

[2] L. Adamczyk, et al., Technical Design Report for the ATLAS Forward Proton Detector, CERN-LHCC-2015-009, ATLAS-TDR-024 (2015), https: //cds . cern . ch/record/2017378. 
[3] K. Cerny, The ATLAS Forward Proton Time-of-Flight Detector System, Vertex 2019: 28th International Workshop on Vertex Detectors, Lopud Island, Croatia, 13 - 18 Oct 2019, https://twiki .cern.ch/twiki/bin/view/AtlasPublic/ForwardDetPublicResults.

[4] L. Nozka, et al., Design of Cherenkov bars for the optical part of the time-of-flight detector in Geant4, Opt. Express 22 no. 23, (2014) 28984-28996.

[5] L. Nozka, et al., Construction of the optical part of a time-of-flight detector prototype for the AFP detector, Opt. Express 24 no. 24, (2016) 27951-27960.

[6] J. Lange, et al., Beam tests of an integrated prototype of the ATLAS Forward Proton detector, JINST 11 (2016) P09005.

[7] L. Chytka, et al., Timing resolution studies of the optical part of the AFP Time-of-flight detector, Opt. Express 26 no. 7, (2018) 8028-8039.

[8] J. Lange, et al., Gain and time resolution of $45 \mu \mathrm{m}$ thin Low Gain Avalanche Detectors before and after irradiation up to a fluence of $10^{15} \mathrm{neq} / \mathrm{cm}^{2}$, JINST 12 (2017) P05003.

[9] T. Komarek, et al., Timing resolution and rate capability of Photonis miniPlanacon XPM85212/A1-S $M C P-P M T$, submitted to NIM A, in the review process.

[10] E. Antamanova et al., Anode current saturation of ALD-coated Planacon ${ }^{R}$ MCP-PMTs, JINST 13 (2018) T09001.

[11] Y. Melikyan, et al., Load capacity and recovery behaviour of ALD-coated MCP-PMTs, Nucl. Instrum. Meth. A 949 (2020) 162854.

[12] L. Nozka, et al., Performance studies of new optics for the time-of-flight detector of the AFP project, submitted to Optics Express, in the review process. 